
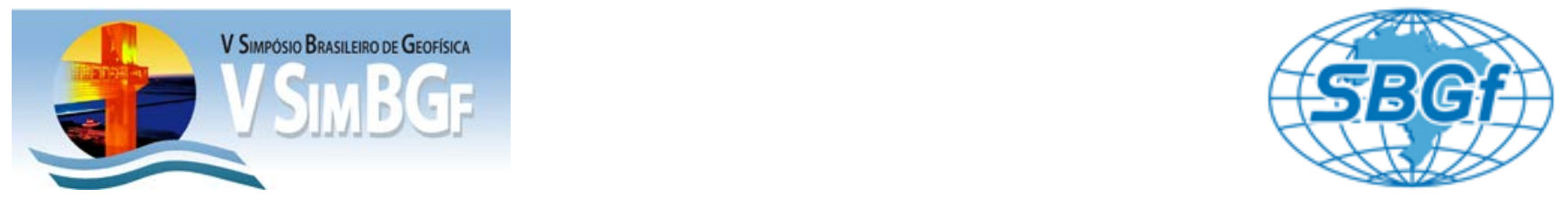

\title{
Decaimento da aceleração de ondas sísmicas na borda da Bacia Potiguar
}

Renato Ramos da Silva Dantas Ł $^{1^{1}}-$ rrsdantas@gmail.com

Joaquim Mendes Ferreira ${ }^{2,3}$, Aderson Farias do Nascimento ${ }^{2,3}$, Eduardo Alexandre Santos de Menezes ${ }^{2}$, Regina Pinheiro

Spinelli' ${ }^{2}$, Neymar Pereira da Costa'.

${ }^{1}$ Curso de Geofísica-UFRN

${ }^{2}$ Departamento de Geofísica-UFRN

${ }^{3}$ PPGG-UFRN

Copyright 2012, SBGf - Sociedade Brasileira de Geofísica

Este texto foi preparado para a apresentação no $V$ Simpósio Brasileiro de Geofísica Salvador, 27 a 29 de novembro de 2012. Seu conteúdo foi revisado pelo Comitê Técnico do $V$ SimBGf, mas não necessariamente representa a opinião da SBGf ou de seus associados. É proibida a reprodução total ou parcial deste material para propósitos comerciais sem prévia autorização da SBGf.

\section{Resumo}

Graças ao projeto RSISNE (PETROBRAS/ UFRN/ FUNPEC), foi possível obter dados de acelerometria de tremores das áreas sísmicas de João Câmara e Pedra Preta, na borda da Bacia Potiguar, nordeste do Brasil, uma das regiões sísmicas mais ativas do país. Com esses dados, buscou-se obter um modelo de decaimento da aceleração de pico (PGA) horizontal nessa região. O modelo utilizado é uma simplificação do modelo de Toro et al. (1997), e o método de regressão múltipla utilizado foi o de mínimos quadrados lineares.

\section{Introdução}

A região da Bacia Potiguar é a área de maior atividade sísmica no país, constando, inclusive, nos mapas globais de perigo sísmico. Apesar disso, pouco se sabe sobre o efeito que um possível tremor, de determinada magnitude e ocorrido em determinado local, teria em dada estrutura construída nessa região. Para isso, necessita-se de registros de aceleração do solo devido a esses tremores. Ou seja, a solução desse problema exige a ocorrência de tremores e a existência de acelerógrafos aptos a registrálos.

Recentemente, o projeto RSISNE (PETROBRAS/UFRN/FUNPEC) permitiu a compra e instalação, no Nordeste do Brasil, de estações permanentes completas (sensor broadband e acelerógrafo), bem como de acelerógrafos individuais que podem ser instalados em áreas sísmicas. Parte dessas estações e acelerógrafos individuais foi instalada na borda da Bacia Potiguar (Figura 1).

Outro fator que possibilitou este trabalho foi a ocorrência de tremores de magnitude razoavelmente grandes (acima de 1.5) nessa região, durante o período de funcionamento das estações instaladas, em particular, nas regiões sísmicas de João Câmara e Pedra Preta. Esses tremores foram registrados, principalmente, nas estações de ACJC e ACMT (ambas na região de João Câmara), ACCP (próxima à área epicentral de Pedra Preta), NBPV (Pedro Velho) e NBPA (Paraú). Como mostra a Figura 1, esse número de estações e sua distribuição espacial proporciona uma boa variabilidade de distâncias epicentrais, fator importante para a construção de um modelo de decaimento.

O objetivo deste trabalho é verificar como se dá o decaimento da aceleração nessa região utilizando-se os dados coletados desde 2011 até agora.

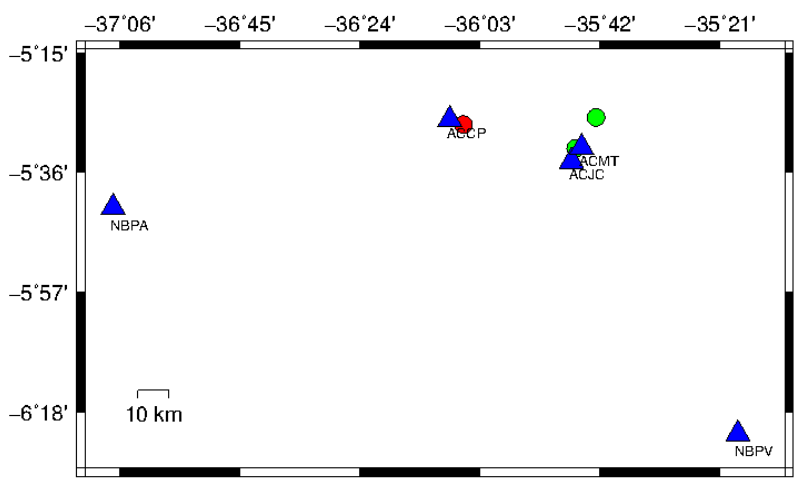

Figura 1: Mapa ilustrando a distribuição das estações utilizadas (NBPA, NBPV, ACCP, ACMT e ACJC) no espaço. O círculo vermelho representa o epicentro médio da área de Pedra Preta (2010); os círculos verdes representam os epicentros da área de João Câmara (2011).

\section{Metodologia}

O primeiro passo para a realização deste trabalho foi a construção de um banco de dados de eventos sísmicos de magnitude $(\mathrm{M})$ conhecida e tal que $\mathrm{M}>1.5$, de área epicentral conhecida e inserida na área investigada (borda da Bacia Potiguar). Nessas condições estão os sismos registrados no período de outubro de 2011 até julho de 2012, totalizando 37 registros de 17 eventos pois, antes de outubro de 2011, não ocorreram sismos de magnitude suficiente para serem registrados nas diversas estações. Esses eventos foram procurados nos acelerogramas (Figura 2) registrados pelas estações utilizadas (ACCP, ACJC, ACMT, NBPV e NBPA). Em seguida, cada registro foi analisado: foram medidos os tempos de chegada das fases $P\left(t_{P}\right)$ e $S\left(t_{s}\right)$ e a amplitude máxima da aceleração (PGA) em cada uma das três componentes (N-S, E-W e Z). A aceleração horizontal $\left(Y_{H}\right)$ foi calculada a partir das componentes Norte-Sul $\left(Y_{N S}\right)$ e Leste-Oeste $\left(Y_{E W}\right)$ usando a equação:

$$
Y_{H}=\left(Y_{N S}^{2}+Y_{E W^{2}}\right)^{0.5}
$$

A leitura das fases $\mathrm{P}$ e $\mathrm{S}$ foram utilizadas para calcular a distância hipocentral $(R)$ de cada evento em relação a cada estação, utilizando o modelo de Terra de semiespaço infinito homogêneo, em que o meio tem 
velocidade de propagação da onda $P\left(v_{P}\right)$ e razão $v_{P} / v_{S}$ $=\mathrm{k}$ tais que $\mathrm{v}_{\mathrm{p}} /(1-\mathrm{k})=8.7$.

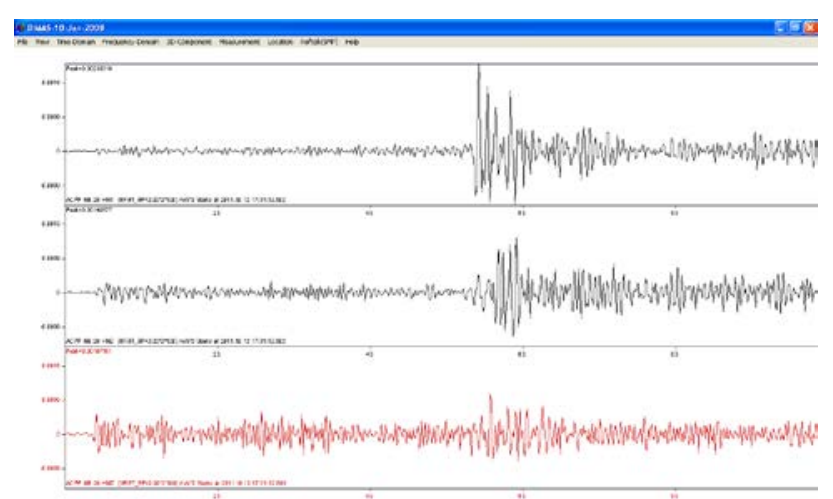

Figura 2: Exemplo de registro acelerográfico. Registro do tremor de João Câmara-RN do dia 12/10/2011 (magnitude 2.3) na estação ACCP (Pedra Preta).

A Figura 3 mostra a distribuição dos registros em relação aos parâmetros magnitude e distância. Visualmente, conclui-se que, na prática, não há correlação entre esses parâmetros.

Em seguida, procurou-se obter o modelo de atenuação para a área investigada. O modelo adotado foi uma equação modificada do modelo de Toro et al. (1997), que leva em conta tanto a magnitude como os efeitos de atenuação com a distância(espalhamento geométrico e absorção). A dispersão não é levada em consideração devido ao fato de que apenas distâncias menores do que 200 km são utilizadas neste estudo. Assim, o modelo utilizado foi:

$$
\ln Y=c_{1}+c_{2} M+c_{3} R+c_{4} \ln R+\varepsilon
$$

Em que $c_{1}, c_{2}, c_{3}$ e $c_{4}$ são constantes a determinar e que variam de região para região, $\varepsilon$ é uma variável aleatória de média zero e variância $\sigma^{2}$, a aceleração $Y$ está em $\mathrm{cm} / \mathrm{s}^{2}$, a distância hipocentral $\mathrm{R}$ em $\mathrm{km}$ e a magnitude $\mathrm{M}$ está na escala regional de Assumpção (1983), mR . Tanto a aceleração de pico vertical $\left(Y_{Z}\right)$ como a horizontal $\left(Y_{H}\right)$ foi utilizada, de modo a comparar os parâmetros do modelo para ambos os casos.

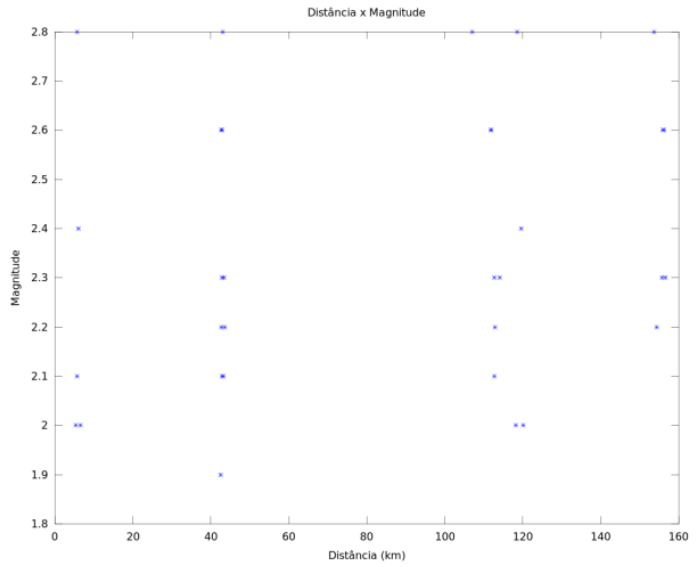

Figura 3: Distribuição dos registros com relação à distância e magnitude.
Por fim, os dados foram ajustados ao modelo a partir de uma regressão múltipla por mínimos quadrados simples, usando o programa OriginPro.

\section{Resultados e Discussão}

A Tabela 1 mostra os parâmetros do modelo obtidos com a regressão, tanto para o decaimento da componente vertical como o da componente horizontal da aceleração de pico. Substituindo os parâmetros ajustados da Tabela 1 na equação (1), chegamos às equação de decaimento para a componente horizontal, com $\mathrm{R}^{2}$ ajustado $=0,93$ :

$\ln Y_{H}=(1,02 \pm 0,96)+(1,34 \pm 0,41) M-(0,012 \pm 0,005) R$

$-(1,28 \pm 0,23) \ln R$

E para a componente vertical, com $\mathrm{R}^{2}$ ajustado $=0,75$ :

$\ln Y_{Z}=(-0,3 \pm 1,0)+(1,35 \pm 0,44) M-(0,006 \pm 0,005) R-$ $(1,16 \pm 0,25) \ln R$

Tabela 1: Coeficientes ajustados ao modelo descrito na equação (1) para cada componente (horizontal e vertical).

\begin{tabular}{|c|c|c|c|c|c|}
\hline Comp. & $\mathbf{C 1}$ & $\mathbf{C 2}$ & $\mathbf{C 3}$ & $\mathbf{C 4}$ & $\boldsymbol{\sigma}$ \\
\hline Vertical & 1,02 & 1,34 & $-0,012$ & $-1,28$ & 0,66 \\
\hline Horizontal & $-0,3$ & 1,35 & $-0,006$ & $-1,16$ & 0,76 \\
\hline
\end{tabular}

Levando-se em conta o método de regressão utilizado (mínimos quadrados simples) e o tamanho da amostra disponível (apenas 37 registros), nota-se que foram obtidos bons ajustes para uma primeira abordagem.

Utilizando as equações de decaimento obtidas, podemos construir curvas que representem graficamente a relação de $Y_{H}$ e $Y_{Z}$ com a distância (Figuras 4 e 5). No mesmo plano, também foram as acelerações modeladas com esse modelo para cada tremor utilizado nessa modelagem, separados em quatro conjuntos de magnitude: I) $1,5<\mathrm{M}<1,8$; II) $1,9<\mathrm{M}<2,2$; II) $2,3<\mathrm{M}<$ 2,6; e IV) $2,7<M<3,0$. Observa-se nessas figuras 0 razoável ajuste obtido com essa metodologia.

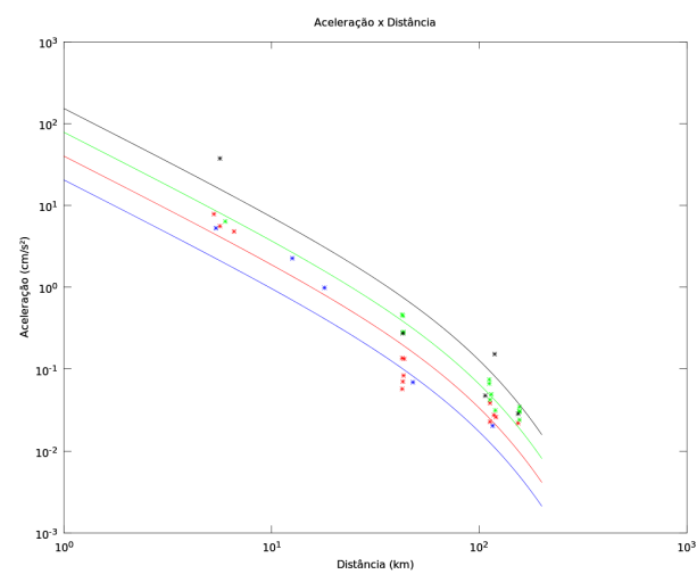

Figura 4: Curvas de decaimento da aceleração horizontal com a distância, para as magnitudes de 1.5 (azul), 2.0 (vermelho), 2.5 (verde) e 3.0 (preto). A dispersão representa os dados experimentais, separados em quatro conjuntos de diferentes faixas de magnitude: 1.5-1.8 
(azul), 1.9-2.2 (vermelho), 2.3-2.6 (verde) e 2.7-3.0 (preto).

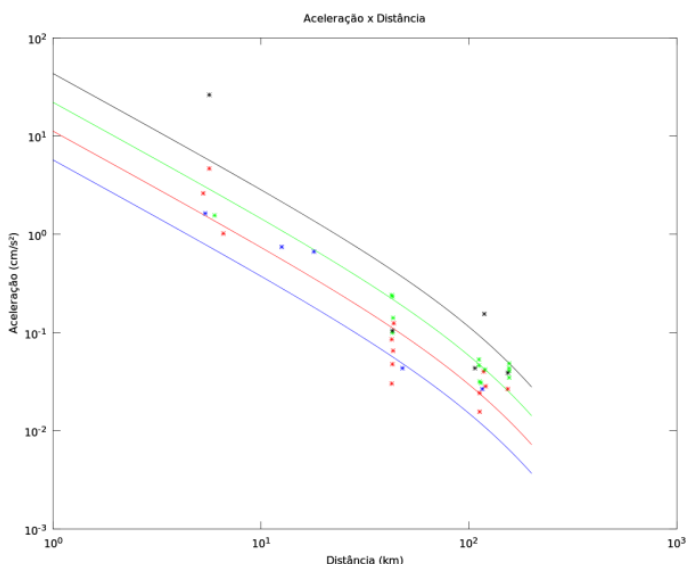

Figura 5: Curvas de decaimento da aceleração vertical com a distância, para as magnitudes de 1.5 (azul), 2.0 (vermelho), 2.5 (verde) e 3.0 (preto). A dispersão representa os dados experimentais, separados em quatro conjuntos de diferentes faixas de magnitude: 1.5-1.8 (azul), 1.9-2.2 (vermelho), 2.3-2.6 (verde) e 2.7-3.0 (preto).

\section{Conclusão}

Enfim, foi possível começar a entender a atenuação das ondas sísmicas na Província Borborema a partir de dados acelerográficos obtidos in situ. O modelo obtido, tanto para a componente horizontal como para a vertical, possui erros razoáveis, levando-se em conta o pequeno número de dados disponíveis e o método de regressão utilizado (mínimos quadrados simples).

Recomenda-se, para trabalhos futuros, construir um novo modelo de decaimento para a Província Borborema, com um maior volume de dados, um modelo mais completo (por exemplo, o modelo original de Toro et al., 1997) e um método de regressão mais adequado às características desse problema (o método apresentado por Joyner \& Boore, 1993, por exemplo, separa as incertezas da estimativa em duas componentes: uma delas relacionada a cada evento e outra relacionada a cada registro).

\section{Agradecimentos}

RRSD agradece à ANP pela bolsa de iniciação científica. JMF e AFN agradecem ao CNPq pelas bolsas de PQ. Este trabalho foi financiado pelo projeto RSISNE (Petrobras/UFRN/FUNPEC) e pelo INCT de Estudos Tectônicos (CNPq/MCT).

\section{Referências}

Assumpção, M., 1989. A regional magnitude scale for Brazil. Bull. Seism. Soc. Am., vol. 73, 1: 237-246.

Domingos, D. O., 2010. Determinação de uma relação preliminar de atenuação sísmica para a Província Borborema (Nordeste do Brasil). Monografia, Instituto de
Astronomia, Geofísica e Ciências Atmosféricas, Universidade de São Paulo, Brasil.

Ferreira, J.M., Oliveira, R.T., Takeya, M.K. \& Assumpção, M., 1998. Superposition of local and regional stresses in northeast Brazil: evidence from focal mechanisms around the Potiguar marginal basin. Geophys. J. Int., 134: 341355.

Joyner, B. W. \& Moore, D. M., 1993. Methods for regression analysis of strong-motion data. Bull. Seism. Soc. Am., vol. 83, 2: 469-487.

Originpro Evaluation, versão 8.6.0, Origin Lab Corporation, USA, 1991-2012.

Toro, G. R., Abrahamson, N. A. \& Schneider, J. F., 1997. Model of strong ground motions from earthquakes in Central and Eastern North America: best estimates and uncertainties. Seismological Research Letters, vol. 68, 1: 41-57. 